\title{
Beam Breakup and the Conceptual Design of Guided and Unguided Accelerators
}

\section{DISCLAIMER}

This report was prepared as an account of work sponsored by an agensy of the United States Government. Neither the United States Government nor any agency thereof, nor ony of their employes, makes any warranty, express or implied, or assumes any legal liability or responsibility for the accuracy, completeness, or usefulness of any information, apparatus, product, or process disclosed, or represents that its use world not infringe privately owned rights. J:eference herein to any specific commercial product, process, or service by trade name, trademark. manufacturer, or otherwise does not necessarily constitute or imply its endorsement, recom. mendation, or favoring by the United States Government or any agency thereof. The views and opinions of euthors expressed herein do not necessarily state or teflect those of the United States Govemment of any agency thereof.

\section{George J. Caporaso}


- Off axis beam entering accelerator gap excites dipole $(M=1) T M$ mode

- Mode has an $E_{z}$ that can extract energy from the beam

- Mode has a transverse $\vec{B}$ that gives the beam an oscillating transverse impulse

- Oscillating transverse impulse develops into transverse displacement in $B_{z}$ field between gaps

- Beam enters next gap with a larger displacement than at previous gap-thus displacement grows 


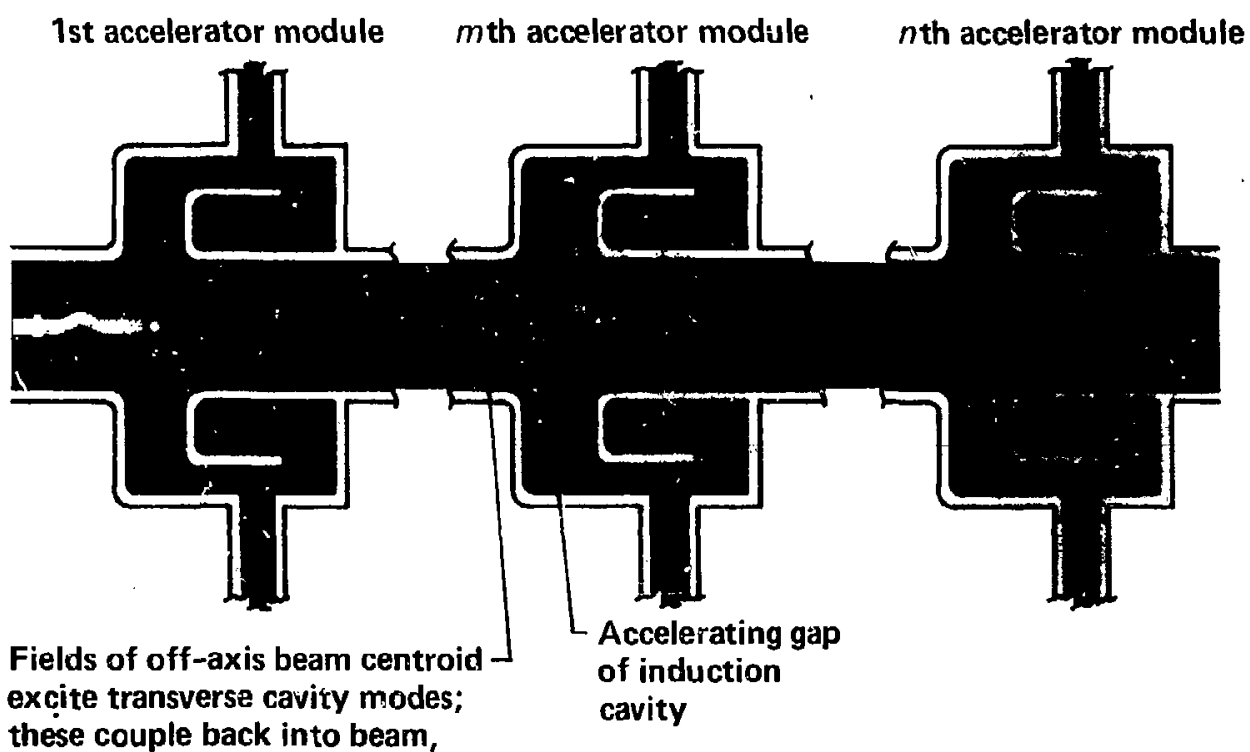
giving it a transvirse "kick" 

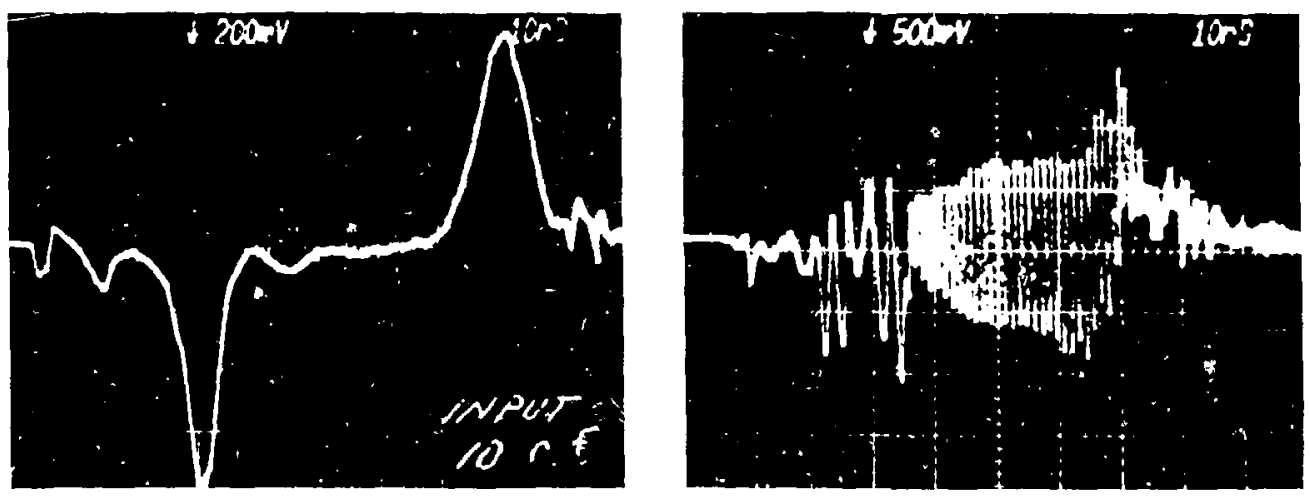

$-4-$

60-B-1938-60 
- Advantages

- Strong focusing

- Anharmonic focusing

- Demonstrated suppression of BBU

- Issues

- Brightness preservation

- Channel formation at high rep rate

- Development of high rep rate laser 


\section{Quadrupole channel option}

- Advantages

- Strong focusing

- No rep rate problems

- Time invariant focusing

- Issues

- Engineering 


\section{P5 PHASE MIX DAMPING OF BEAM OFF-AXIS DISPLACEMENTS}

- Consider a line charge as the focusing field for beam electrons

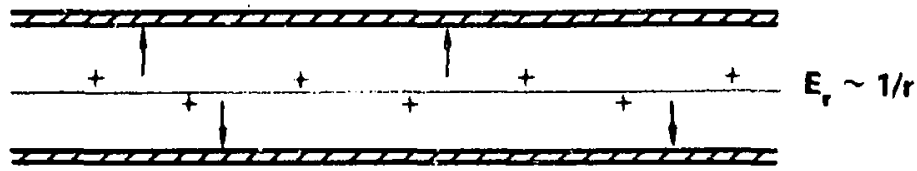

- Beam electrons far off axis will oscillate slower than electrons near ax is

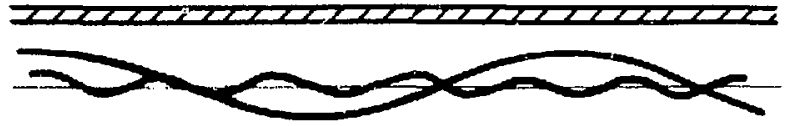

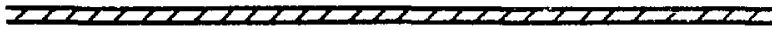

- This "orbitai phase mixing" changes coherent displacements

into random motion (entropy), or increased emittance
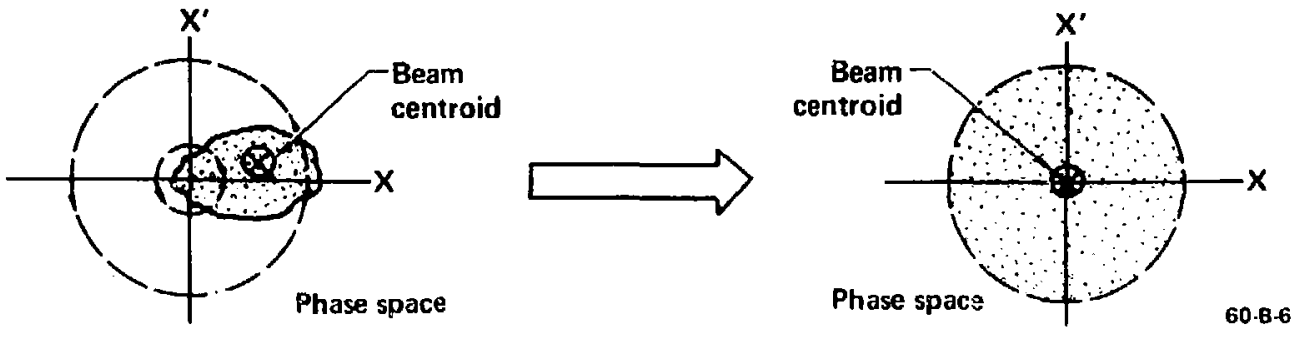
- Future ETA experiments (summer '83) will produce the anharmonic potential by pre-ionizing a preferred channel in a very low pressure gas ( 1 micron)
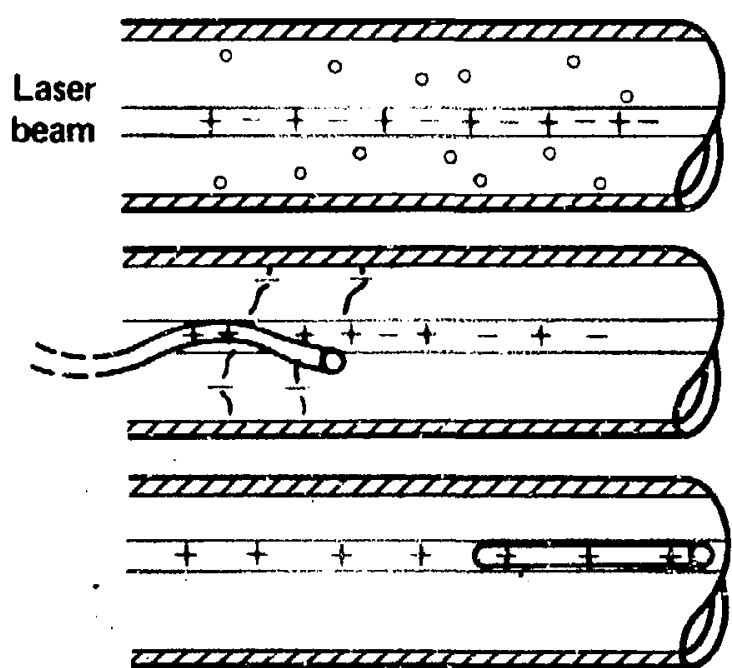

A pre-ionized channel is formed within a circular beam transport pipe

The electron beam ejects secondary electrons and forms a preferred axis ion channel (strongly anharmonic potential)

The off-axis beam is centered and beam oscillations are phase mixed clamped 
Without laser guiding (using conventional magnetic solenoids) growth of transverse beam motion degrades ATA beam

- Oscilloscope traces from small magnetic induction loops located along the accelerator show growth of transverse beam motion that results from the beam coupling to a reasonant $(800 \mathrm{MHz})$ transverse mode of the accelerator modules (al! traces horizontal 10nS/div, vertical arbitrary)

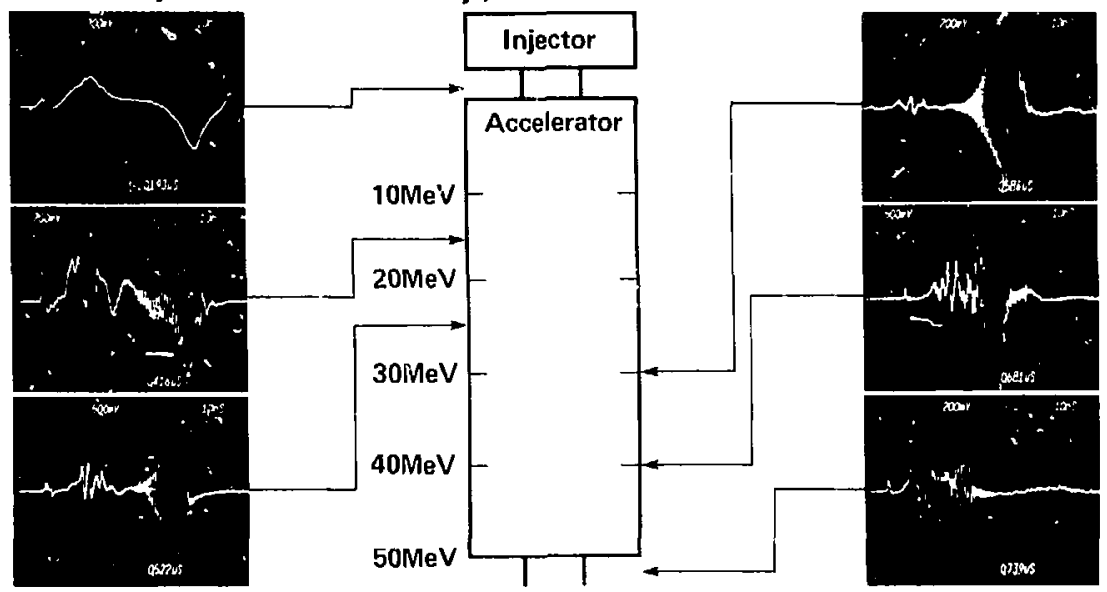




\section{With electrostatic transport transverse beam motion within ATA is stabilized and suppressed}

- Oscilloscope traces on left are from magnetic induction loops at different accelerator locations. Data from one beam pulse is shown in each column. The two columns indicate reproducibility. The oscilloscope trace in the bottom right is accelerator output current ( $2 \mathrm{kA} /$ div. $10 \mathrm{nS} /$ div.).
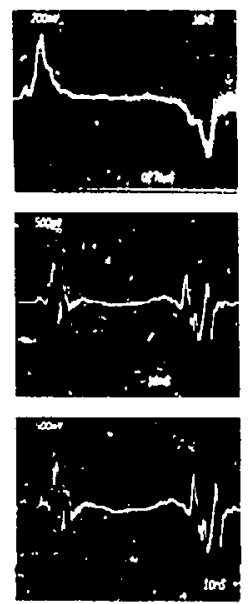

$60-B-1284-23$
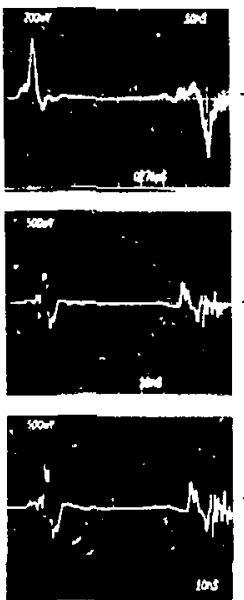

lans

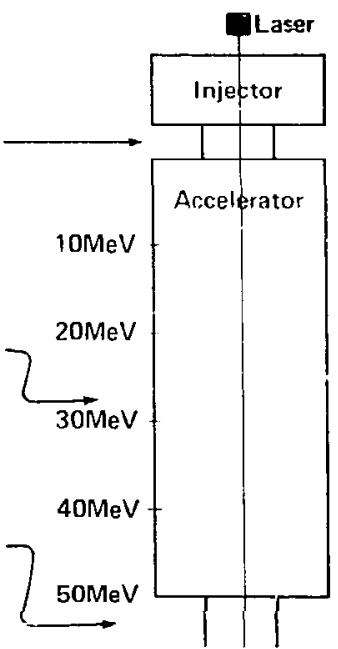

Accelerator output current $2 \mathrm{kA} /$ div, 10nS/div.

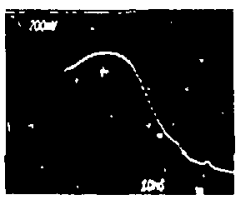


Laser

- Wavelength

- Pulsewidth

- Energy

- Spatial profile

- Divergence

- Centroid jitter

- Repetition rate

- Timing

Benzene

- Pressure

- Flow rate

- Replenishment rate

- Ionization fraction
$249 \mathrm{~nm}$ (KrF)

28 ns FWHM

$0.4 \mathrm{~J}$

$20 \times 12 \mathrm{~mm}, 12 \times 7 \mathrm{~mm}$

$50 \mu \mathrm{r}$

$100 \mu \mathrm{r}$

$0.2-1 \mathrm{~Hz}$

$\sim 950$ ns before e-beam

$0.1-0.5 \mu \mathrm{m}$

$1-6 \mathrm{scc} / \mathrm{min}$

$0.3-2 \mathrm{~Hz}$

$1-3 \%$ 


\section{Difference in ATA operation when beam transport}

\section{and focusing is accomplished by conventional}

\section{solenoids versus laser guiding}

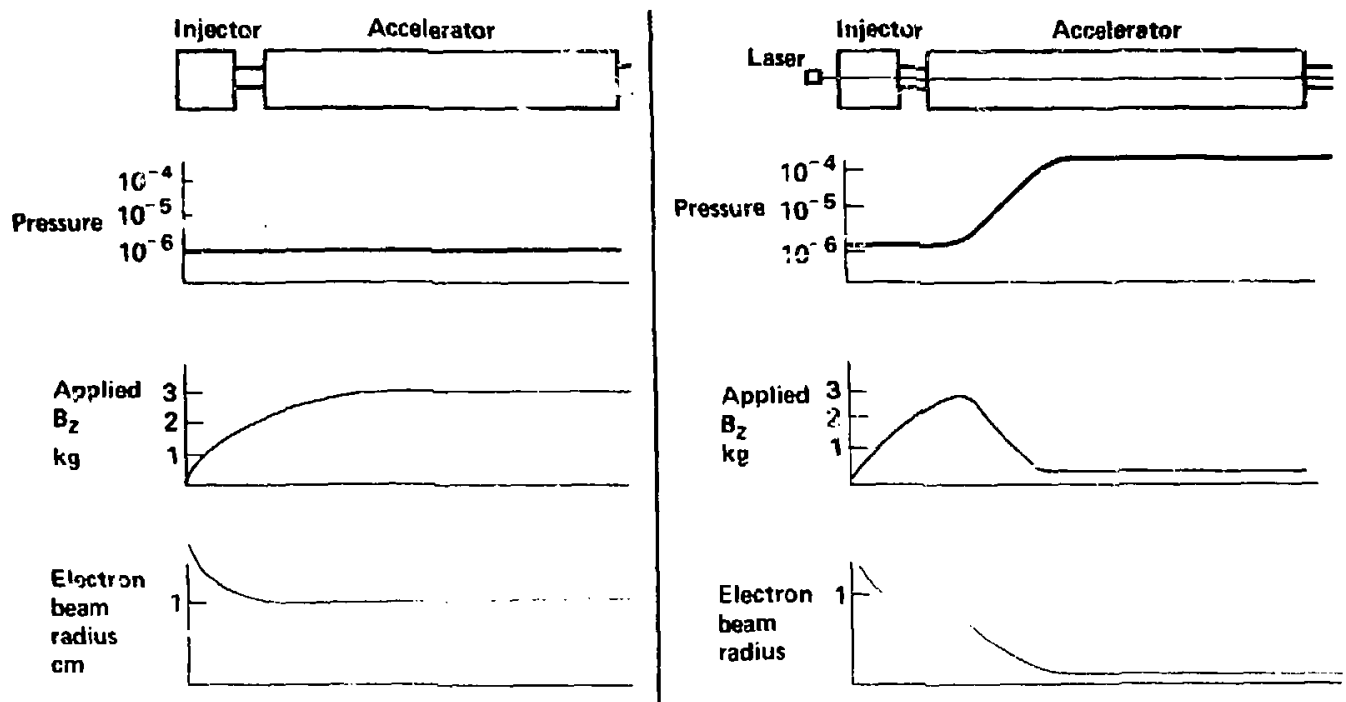




\section{Quadrupole channels}
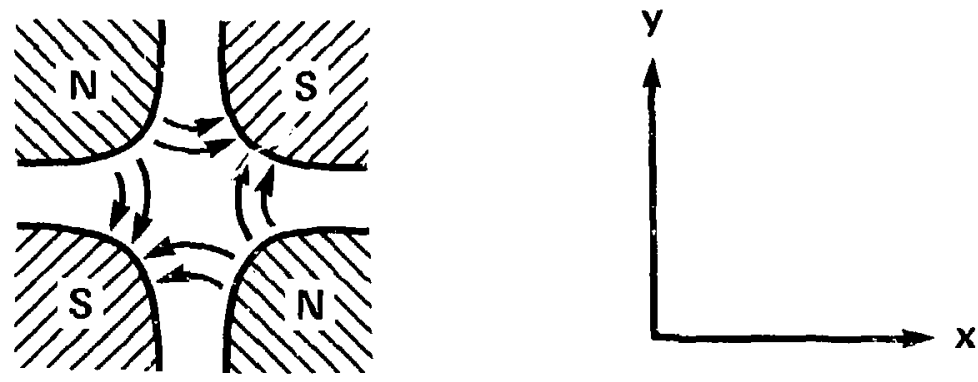

$B_{x}=g y \phi(z)$
$B_{y}=g \times \phi(z)$

$\mathrm{g}$ is the "gradient"

Equations of motion:

$\frac{\partial^{2} y}{\partial z^{2}}+\frac{e g}{\gamma m c^{2}}$ y $\phi(z)=0$

$\frac{\partial^{2} x}{\partial z^{2}}-\frac{e g}{\gamma m c^{2}} \times \phi(z)=0$

For a periodic cinannel fourier analyze $\phi(z)$ 


\section{Alternating singlets}
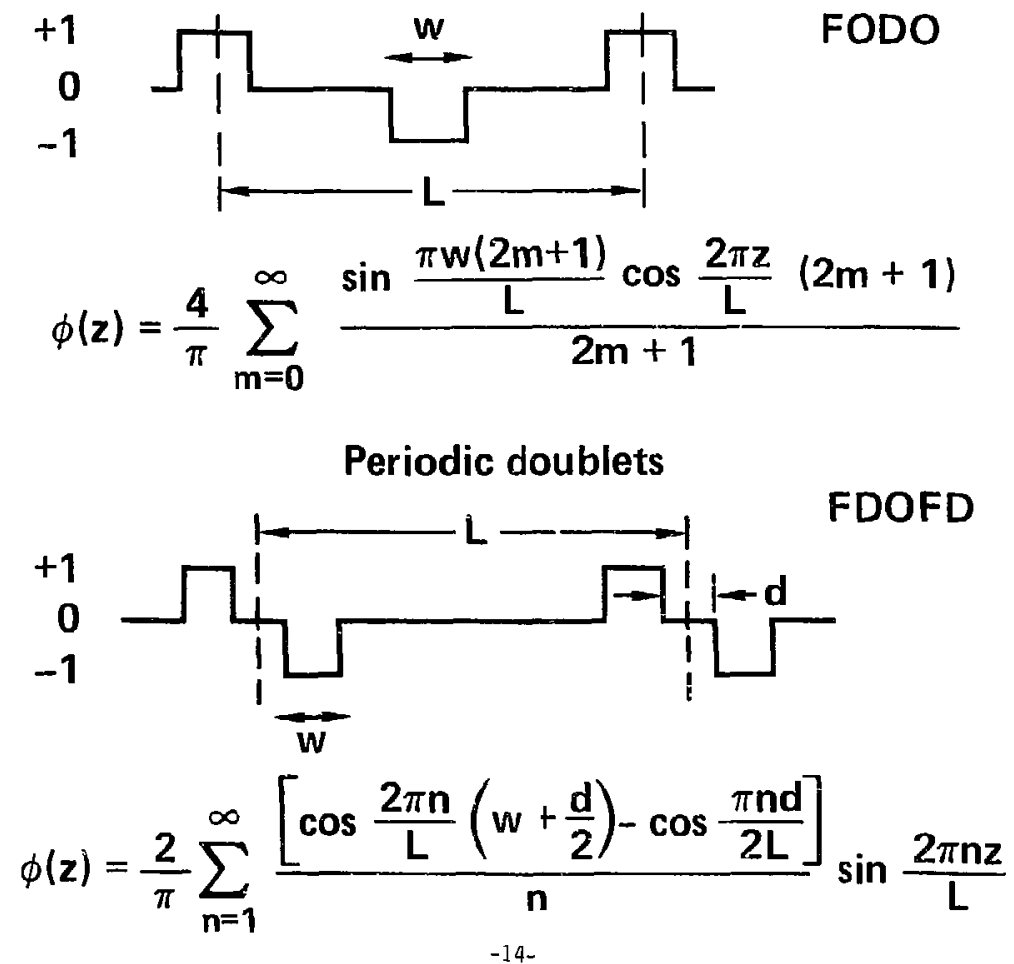


$$
\begin{aligned}
y(z) & =a \cos (\mu x+\theta)\left[1+\epsilon \sum_{n=1}^{\infty} \frac{A n}{h^{2}} \cos n z\right] \\
& =\operatorname{acos}\left(k_{\beta} z+\theta\right)\left[1+\frac{k^{2}}{k_{0}^{2}} \sum_{n=1}^{\infty} \frac{A n}{n^{2}} \cos \frac{2 \pi n z}{L}\right] \\
k_{\beta} & =\mu \epsilon k_{0}=\frac{\mu k^{2}}{k_{0}} \quad \mu^{2}=\frac{1}{2} \sum_{n=1}^{\infty} \frac{A n^{2}}{n^{2}} \\
k_{\beta} & =\frac{\mu}{2 \pi} \frac{e g L}{\gamma m c^{2}}
\end{aligned}
$$

For an FODO lattice

$$
k_{\beta}=\frac{\sqrt{2}}{\pi^{2}} \sin \frac{\pi w}{L}\left[\frac{e g L}{\gamma \mathrm{mc}^{2}}\right]
$$




$$
\begin{aligned}
& {\left[\frac{\partial^{2}}{\partial \tau^{2}}+\frac{\omega_{0}}{\mathbf{Q}} \frac{\partial}{\partial \tau}+\omega_{0}^{2}\right] \Delta=\frac{\omega_{0}^{2} \mathbf{G} \xi}{\gamma}} \\
& \frac{\partial}{\partial \mathbf{z}}\left[\gamma \frac{\partial \xi}{\partial z}\right]+\gamma \mathbf{k}_{\beta}^{2} \xi=\gamma \Delta \\
& \omega_{0}=\text { Angular frequency of mode } \\
& \xi=\text { Beam centroid displacement } \\
& \mathrm{Lg}=\text { Average gap separation } \\
& \Delta=\frac{1}{\operatorname{Lg}}\left[\frac{\Delta \mathbf{p}_{1}}{\mathbf{p}_{\mathbf{z}}}\right] \\
& \mathbf{G}=\frac{\omega_{0}\left(\mathbf{z}_{1} / \mathbf{Q}\right) \mid}{\operatorname{Lg} \mathbf{l}_{0}}
\end{aligned}
$$


Solve model equations by Fourier transform in $\tau$

$$
\begin{aligned}
& \frac{\partial^{2} \widetilde{\Psi}}{\partial z^{2}}+\left[k_{\beta}^{2}-\frac{h(\omega)}{\gamma}\right] \widetilde{\Psi} \cong 0 \\
& \xi(z, \omega)=\frac{\widetilde{\Psi}(z, \omega)}{\sqrt{\gamma}} \\
& h(\omega)=\frac{\omega_{0}^{2} G}{\omega_{0}^{2}-\omega^{2}+\frac{i \omega \omega_{0}}{Q}}
\end{aligned}
$$

For resonant excitation (steady state gain)

$$
\xi \sim \exp \left[-i \int_{0}^{z}\left[k_{\beta}^{2}+\frac{i G Q}{\gamma}\right]^{1 / 2} d z^{\prime}\right]
$$




$$
\xi \sim \exp \left[\mathrm{GQ} \int_{0}^{2} \frac{d z^{\prime}}{2 k_{\beta} \gamma}\right]
$$

For $\gamma(z)=\gamma_{0}+\lambda z$

$$
\begin{array}{ll}
\mathrm{k}_{\beta}=\frac{\mathbf{k}}{\gamma} & \xi \sim \exp \frac{\mathrm{GQ}\left(\gamma-\gamma_{\mathbf{0}}\right)}{2 \mathbf{k} \lambda} \\
\mathrm{k}_{\beta}=\frac{\mathbf{k}}{\sqrt{\gamma}} & \xi \sim \exp \frac{\mathrm{GQ}\left(\sqrt{\gamma}-\sqrt{\left.\gamma_{0}\right)}\right.}{2 \mathrm{k} \lambda} \\
\mathrm{k}_{\beta}=\mathrm{k} & \xi \sim \exp \frac{\mathrm{GQ} \ln \left(\gamma / \gamma_{0}\right)}{2 \mathrm{k} \lambda}
\end{array}
$$




\section{ATA $250 \mathrm{kV}$ induction cell}

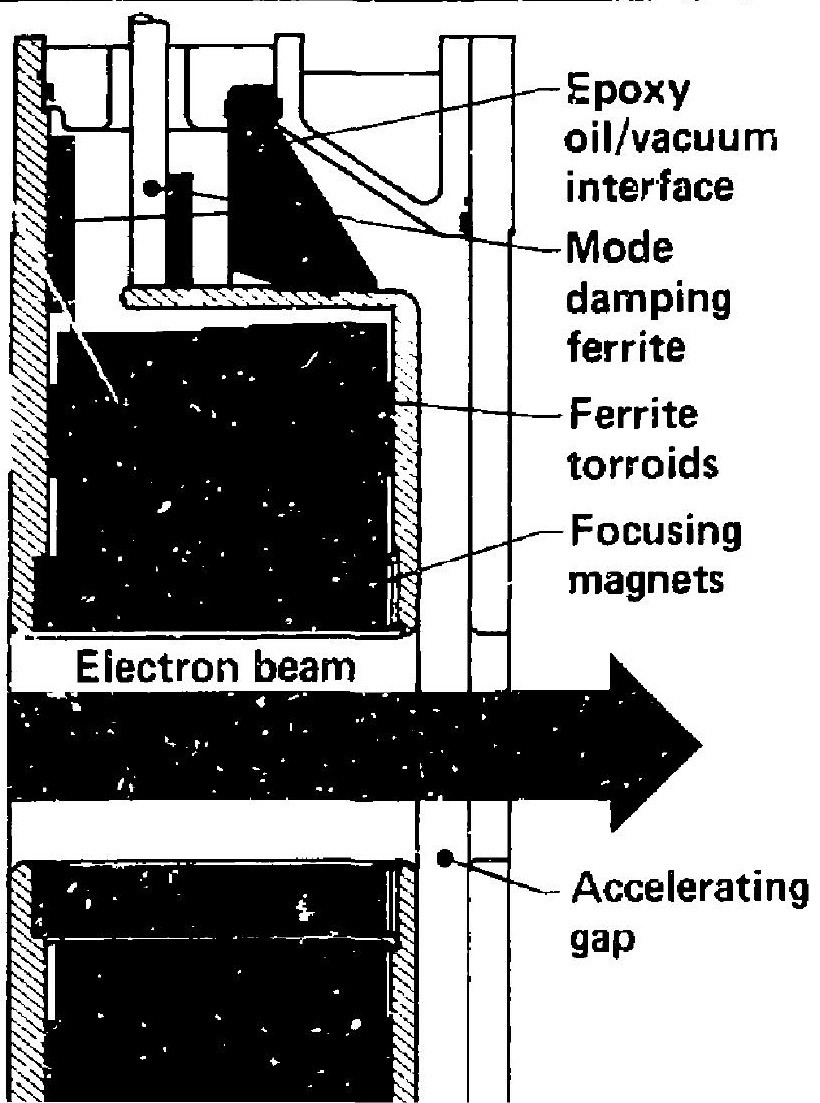


Suriace impedance $Z_{s}$

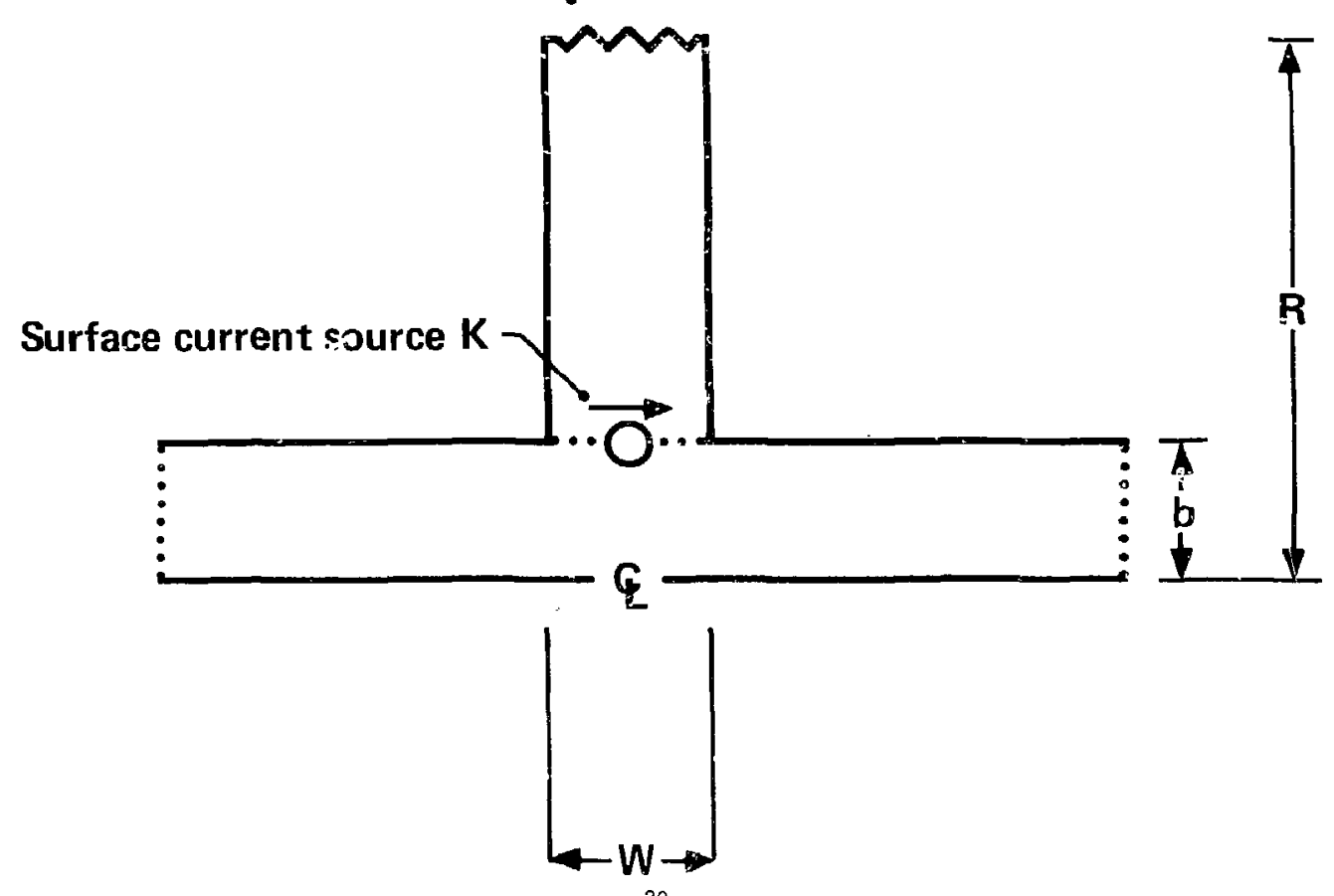

60-B-1085-32 
Response for ATA cavity

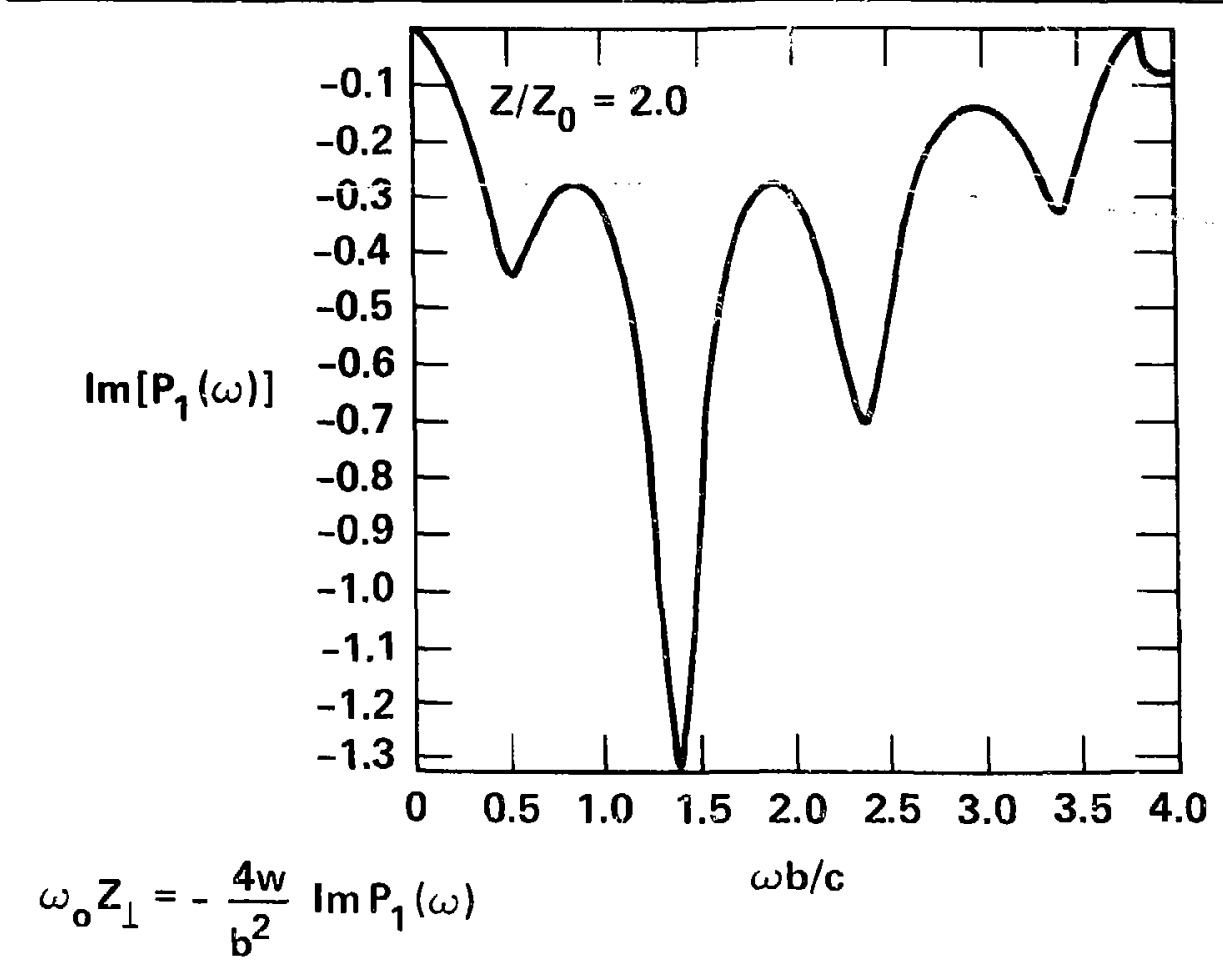

60-B-1085-29 
Results of cavity model

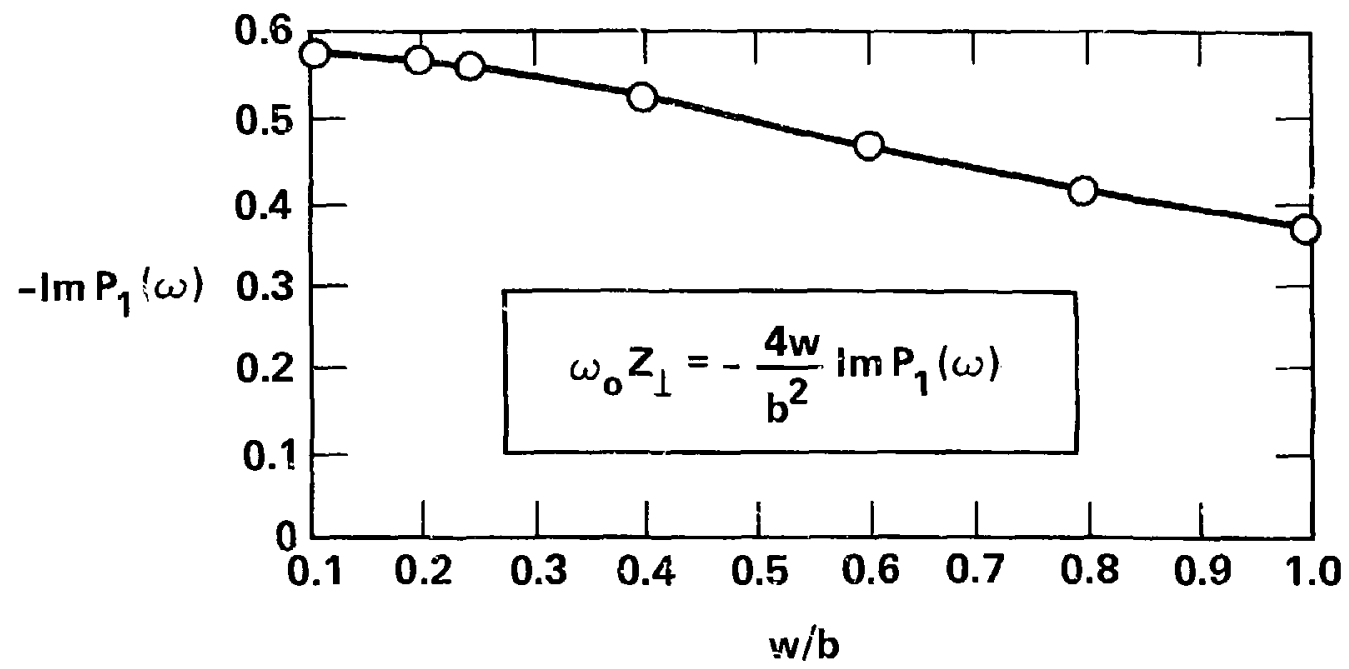

Minimum of the negative imagirary part of the cavity resporse as a function of gap width $w$ over pipe radius $b$ from the cavity model. The quantity $\omega_{0} z_{1}$ is the critical parameter which determines growth of the beam breakup instability 


\section{Proposed accelerator output beam specifications}

\begin{tabular}{ll} 
Current & $=3 \mathrm{kA}$ \\
Energy & $=300 \mathrm{MeV}$ \\
\hline Brightness & $=2 \times 10^{6}$ \\
Emittance & $=0.055 \mathrm{rad}-\mathrm{cm}$, edge \\
Pulse length & $>50 \mathrm{~ns}$ \\
Rep rate & $100 \mathrm{~Hz}$ continuous \\
& $5 \mathrm{kHz}$ for 30 seconds \\
Beam $\Delta \mathrm{X}$ & $\leqslant 0.01 \mathrm{~cm}$
\end{tabular}


Conceptual point design

- Cell coupling (all cells)

$\begin{array}{llrl}\text { I.D. }=5.875 \text { in } & b & =7.48 \mathrm{~cm} \\ \text { Gap }=0.25 \mathrm{in} & w & =0.635 \mathrm{~cm}\end{array}$

$\omega_{0} z_{\perp}=2 \times \frac{4 w}{b^{2}}(0.6)=0.055 \mathrm{~cm}^{-1}$

(ATA value $=0.27 \mathrm{~cm}^{-1}$ )

- Low gamma section: $\gamma_{0}=5$ to $\gamma_{\mathrm{T}}=\mathbf{5 0}$ solenoids

- Tune

$$
\begin{aligned}
& B_{z}=1 \mathrm{kG} \text { at } \gamma_{0}=5 \text { Linearly } \\
& =3 \mathrm{kG} \text { at } \gamma_{F}=15 \text { ramped } \\
& =3 \mathrm{kG} \text { flat up to } \gamma_{\mathrm{T}}=\mathbf{5 0} \\
& \xi=\frac{\xi_{0}}{2} \exp \left[\frac{1}{I_{0}} \frac{\mathrm{mc}^{2}}{\Delta \mathrm{Eg}^{2}} \frac{\omega_{\mathrm{o}} z_{\perp}}{\mathrm{K}_{\mathrm{os}}}\left\{\gamma_{\mathrm{T}}-\gamma_{\mathrm{F}}+\gamma_{\mathrm{F}} \frac{\ln \gamma_{\mathrm{F}}}{\gamma_{\mathrm{o}}}\right\}\right] \\
& \mathrm{K}_{\mathrm{os}}=\frac{\mathrm{eB}}{\mathrm{mc}^{2}} \quad \xi / \xi_{\mathrm{o}}=1.3
\end{aligned}
$$


- Intermediate $\gamma: \quad \gamma_{0}=50$ to $\gamma_{F}=200$

Doublets, FDOFO ....

Tune: $\quad g=0.29 \mathrm{~kg} / \mathrm{cm}\left(B_{\text {pole }}=2.2 \mathrm{~kg}\right)$ at $\gamma_{0}$ $\mathrm{g}=1.16 \mathrm{~kg} / \mathrm{cm}\left(B_{\text {pole }}=8.8 \mathrm{~kg}\right)$ at $\gamma_{F}$

Linearly ramped to give $\lambda_{\beta}=\mathbf{5 . 0}$ meters

$\xi=\frac{\xi_{0}}{2}\left(\frac{\gamma_{F}}{\gamma_{D}}\right)$

$\frac{1}{2}\left[\frac{1}{1} \frac{\omega_{0} z_{\perp}}{k_{p}} \frac{m c^{2}}{\Delta E g}-1\right]$

$$
\xi / \xi_{0}=1.5 \quad f=1.35
$$

- High $\gamma$ section:

$$
\gamma_{\mathrm{o}}=200 \text { to } \gamma_{\mathrm{F}}=588
$$

Singlets, FODO . . .

Tune: $\quad g=0.44 \mathrm{~kg} / \mathrm{cm}\left(B_{\text {pole }}=3.3 \mathrm{~kg}\right)$ at $\gamma_{0}$

$\mathrm{g}=1.28 \mathrm{~kg} / \mathrm{cm}\left(B_{\text {pole }}=9.75 \mathrm{~kg}\right)$ at $\gamma_{F}$

Linearly ramped to give $\lambda_{\beta}=7.0$ meters

$$
\xi / \xi_{o}=2.1 \quad f \cong 1.7
$$




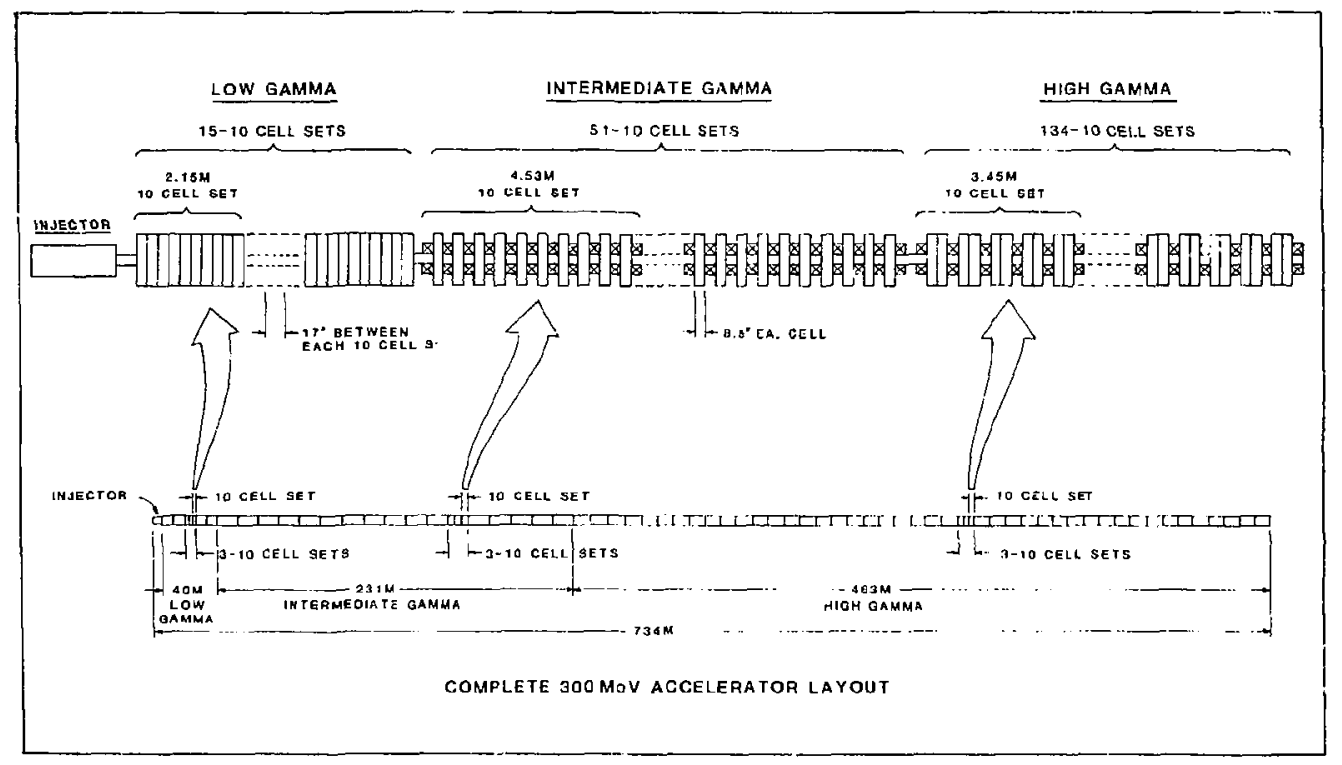

$-25-$ 
Forrite

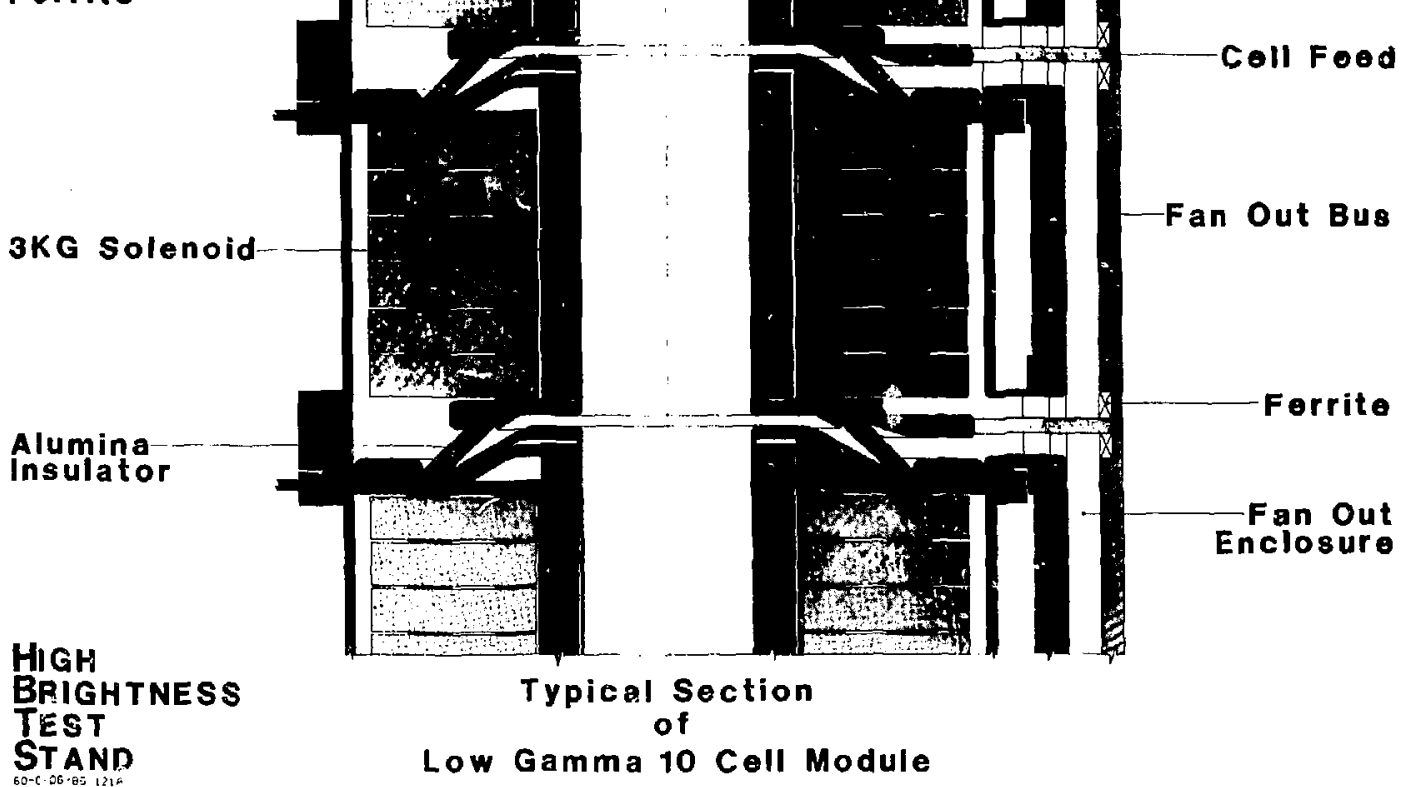


10 cell block - intermediate gamma section

(25 MeV - $100 \mathrm{MeV}$ )

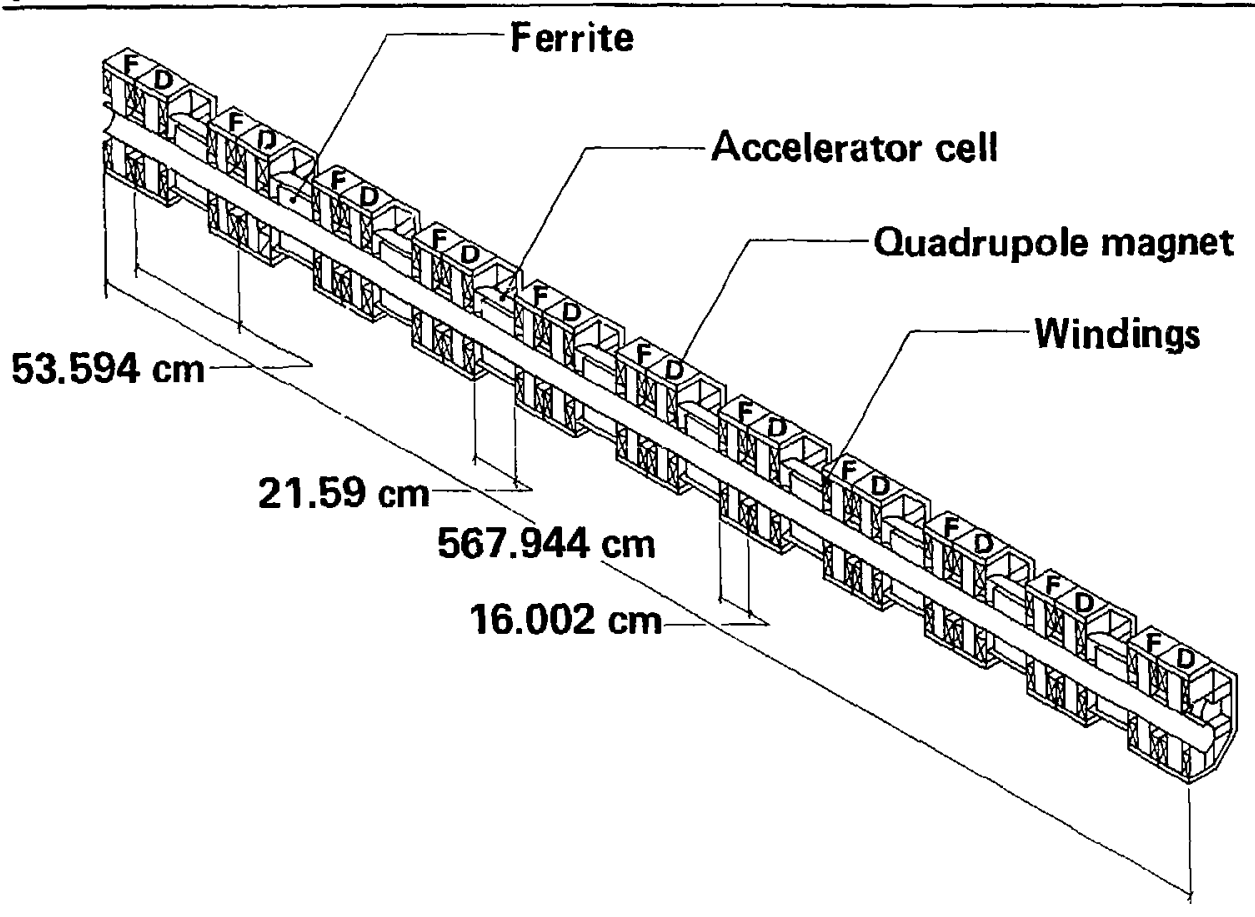




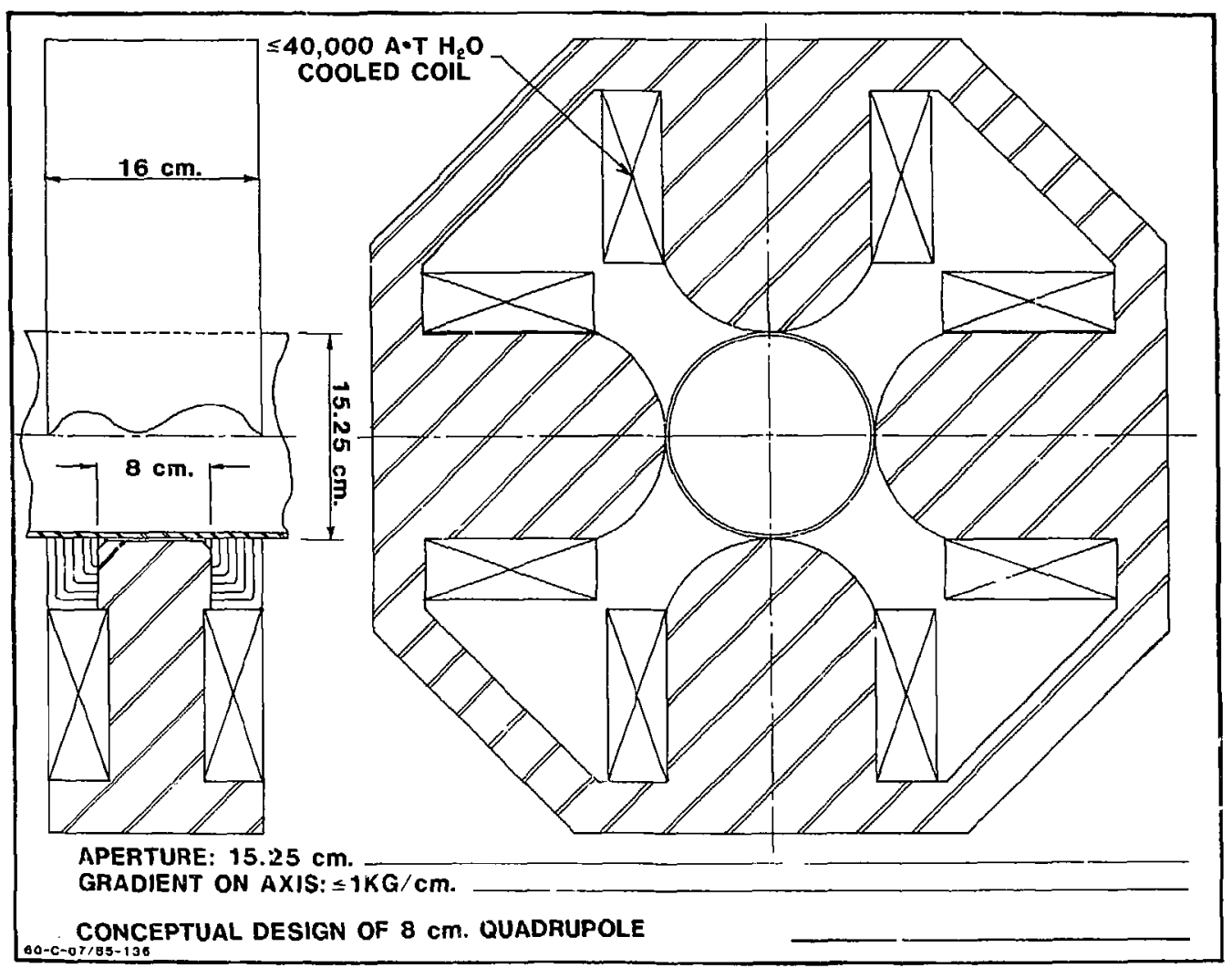

$-29-$ 
$8 \mathrm{~cm}$ doublet

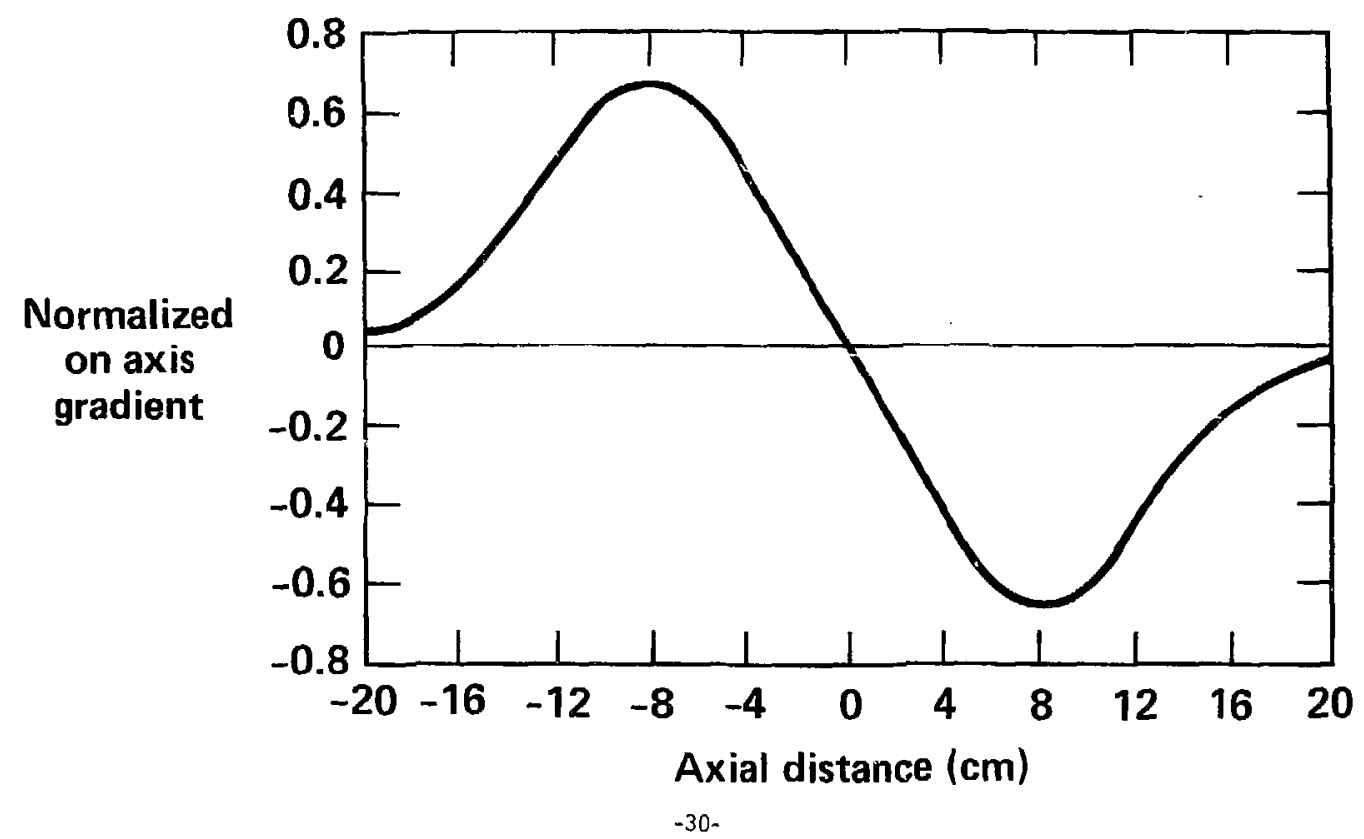

60.C.0785-140 


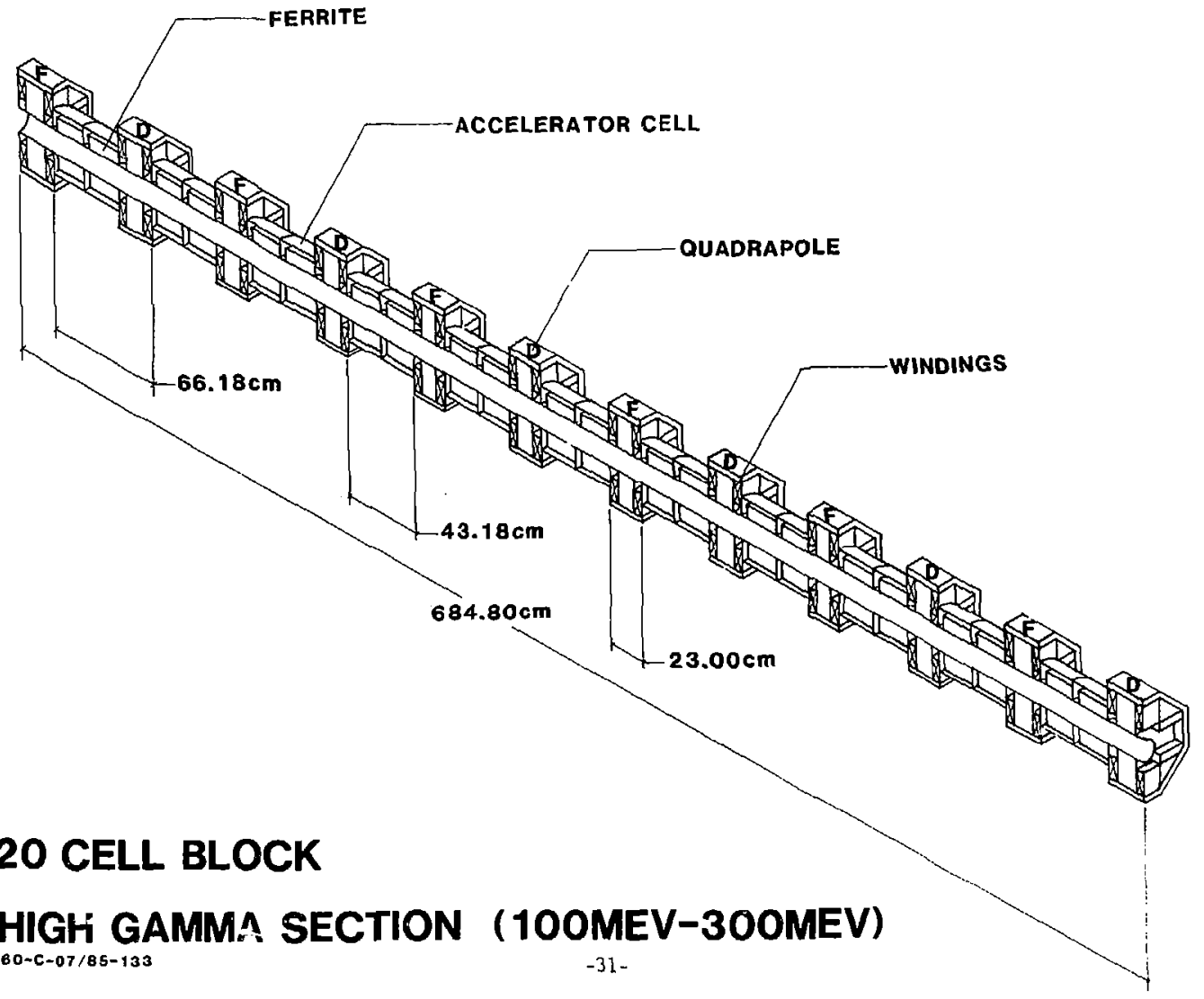




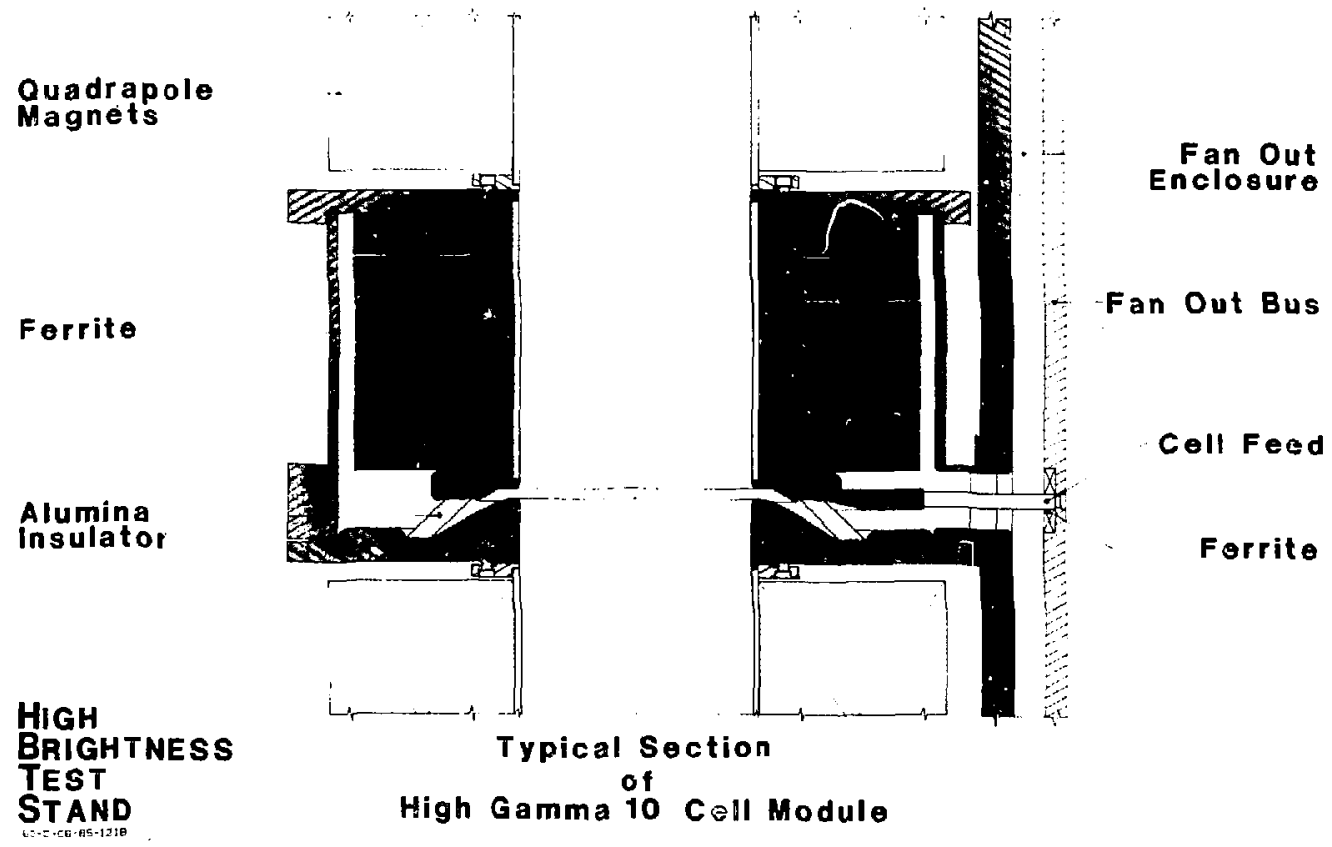




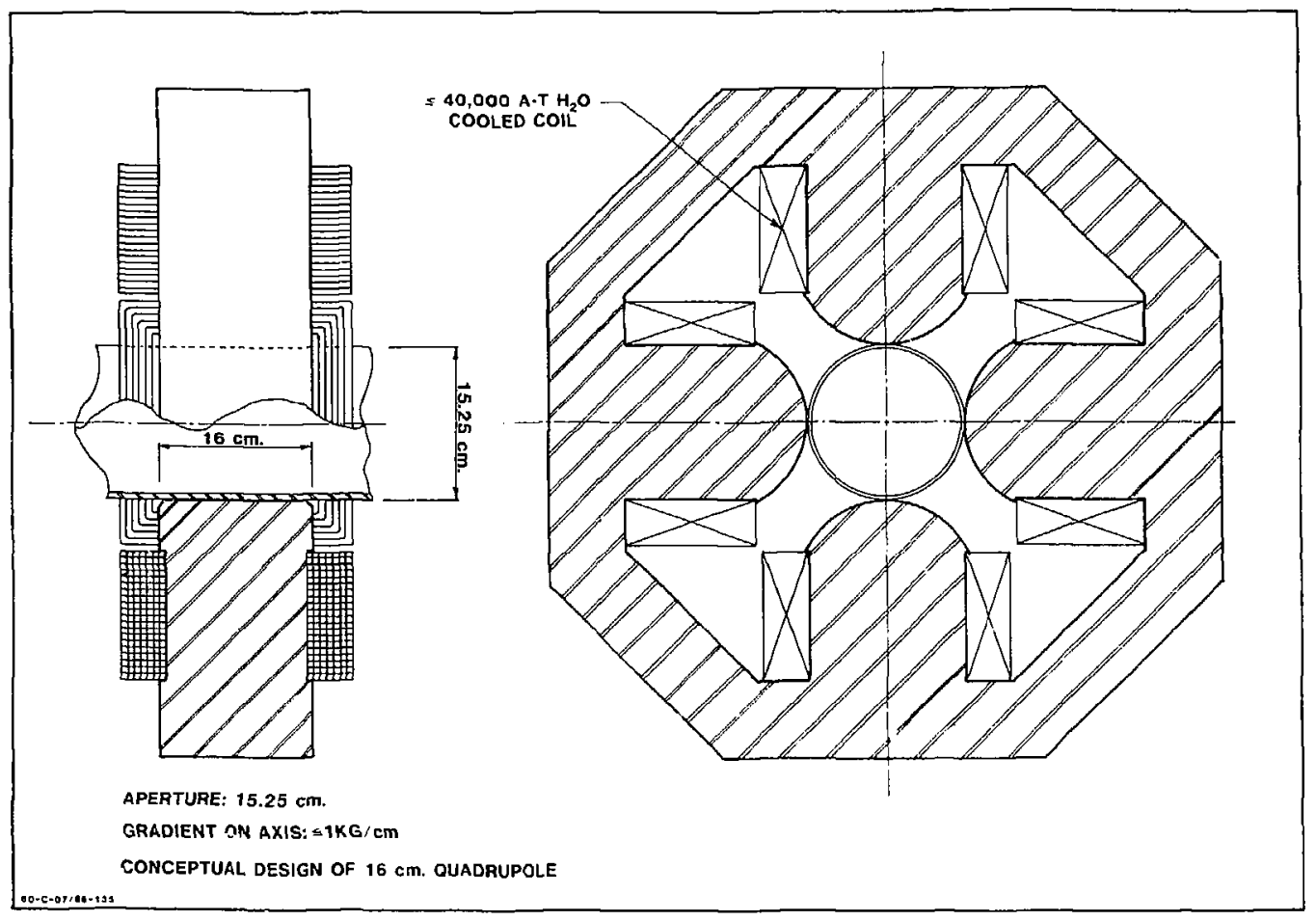


Predicted on axis gradient profile for $16 \mathrm{~cm}$ wide quadrupole

$16 \mathrm{~cm}$ quadrupole

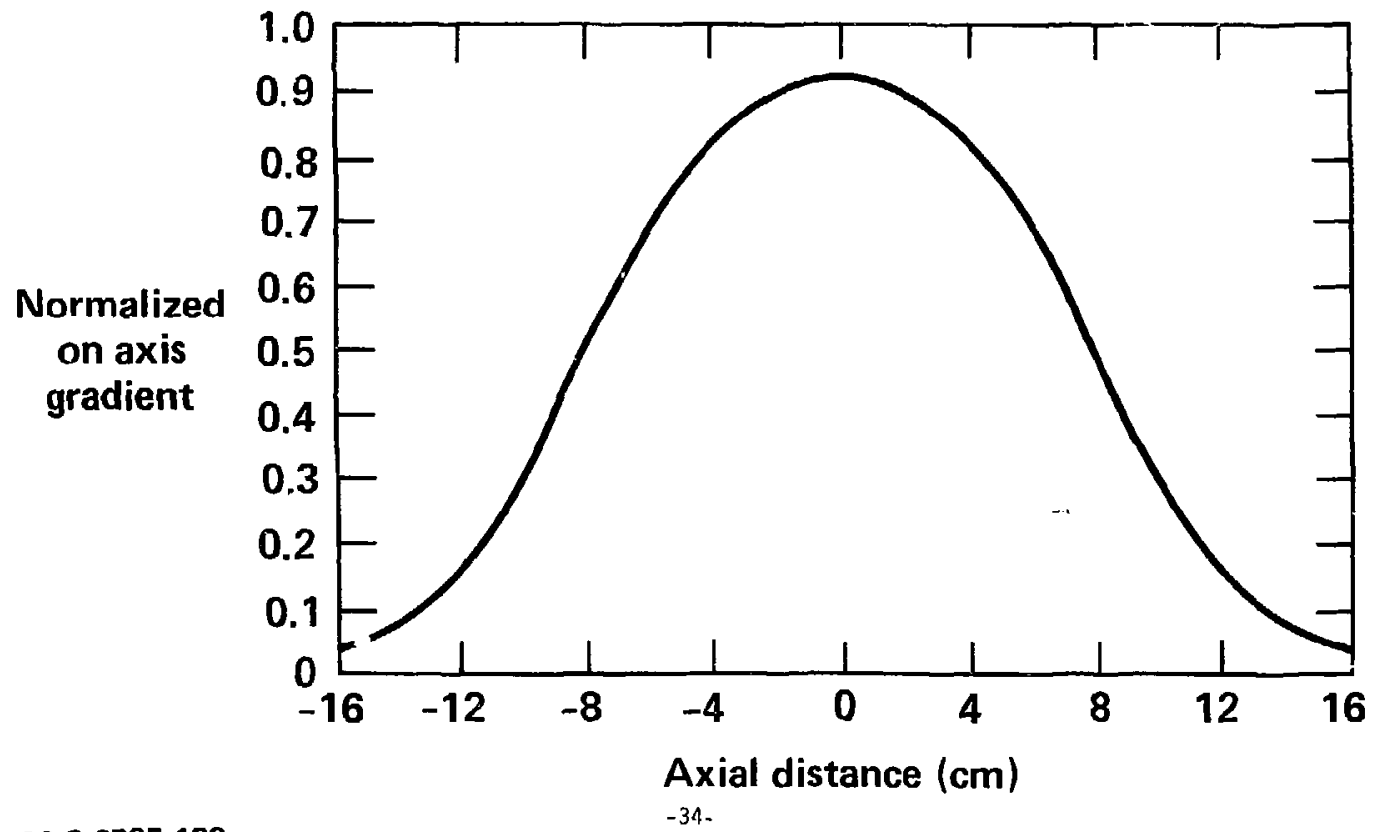

